\title{
Nonlinear multimodal electromagnetic device for vibration energy harvesting
}

\author{
K. Aouali ${ }^{1,2}$, Z. Zergoune ${ }^{1}$, N. Kacem ${ }^{1}$, E. Mrabet ${ }^{2}$, N. Bouhaddi ${ }^{1}$ and M. Haddar $^{2}$
}

1. Univ. Bourgogne Franche-Comté, FEMTO-ST Institute, CNRS/UFC/ENSMM/UTBM, Department of Applied

Mechanics, 25000 Besançon, France.

Email : \{kaouthar.aouali, zakaria.zergoune, najib.kacem, noureddine.bouhaddi\}@ femto-st.fr

2. Univ. Sfax, National Engineering School of Sfax, Research Laboratory of Mechanics Modeling and Production, 3038

$$
\text { Sfax, Tunisia }
$$

Email : elyes.mrabet@yahoo.fr, mohamed.haddar@enis.rnu.tn

\begin{abstract}
:
A multimodal vibration energy harvesting in a periodic system is proposed. The multimodal approach and the nonlinearity are implemented in order to improve the performances of the studied device. The periodic system, based on electromagnetic transduction, consists of two weakly coupled magnets mechanically guided by two elastic beams. The quasi-periodic system is obtained by varying the mass of one of the moving magnets which leads to the vibration energy localization in regions close to the imperfections. This phenomenon is exploited to maximize the harvested energy. The mechanical nonlinearity is introduced by considering large displacements of the beams. The system is modeled by two coupled forced Duffing equations. The governing equations are solved using finite difference method combined with arc-length method. It is shown that the introduction of the nonlinearity leads to the enlargement of the bandwidth and the increase of the amplitude of the vibration.
\end{abstract}

Keywords : Vibration energy harvesting, multimodal method, energy localization, nonlinearity, quasi-periodic structure.

\section{Introduction}

Over the past few years, the diversity of ambient sources of energy arouses researchers to make its scavenging a major challenge [1]. In order to remote systems from renewable power and to replace batteries, many efforts have been developed with the aim of exploiting the omnipresence of vibration energy. Therefore, various techniques based on different conversion mechanisms have been developed [2].

Although the evolution in this domain is continuous, most devices operate on a narrow frequency band, which limits their application in areas where energy prevails over a large frequency band $[1,3]$. To overcome this problem, several approaches have been proposed such as the introduction of nonlinearities [4], the adoption of multimodal configurations [3] and the energy localization [5, 6], etc. In this context, systems generating electricity from ambient vibrations have become a focus of interest of academic and industrial research.

In this work, a multimodal vibration energy harvester (VEH) is proposed. While focusing on the performances improvement, introducing nonlinearity by considering large displacements of beams and perturbing the structure by changing mass of one of the moving magnets [7] is investigated. The nonlinearities and the mistuning introduced in the model allow enlarging the bandwidth and localizing the energy.

\section{System modelling}

\subsection{Design}

The proposed harvester shown in Figure 1 is inspired from the existing work of Mahmoudi et al [4] . It is composed of two weakly coupled magnets guided by elastic beams. Copper coils are wrapped around the moving magnets. A current is induced in coils when magnets oscillate around their equilibrium positions (Lenz' low). This current will be exploited to harvest energy.

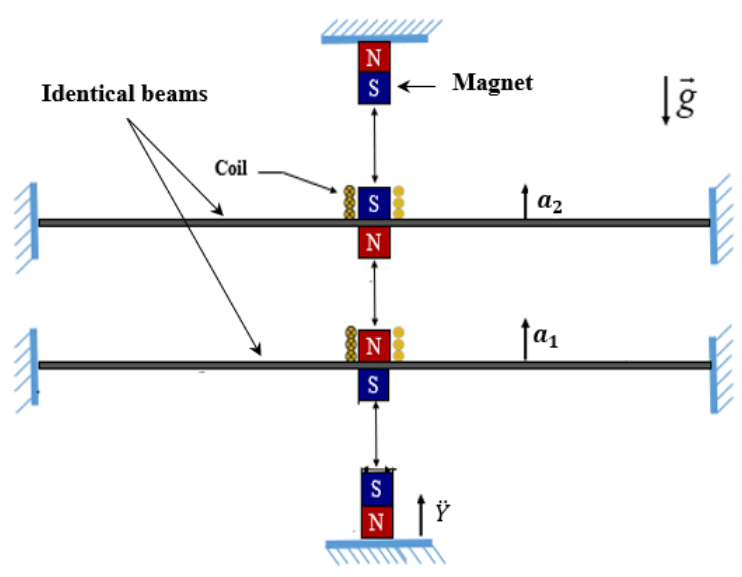

Figure 1. The proposed multimodal VEH device based on electromagnetic transduction 


\subsection{Governing Equations}

The fourth order partial differential equations governing the continuum system can be derived using the Hamilton principle. Then, a reduced-order model can be generated by Galerkin method. The system can be transformed into a finite degree-of-freedom system in terms of generalized coordinates.

We consider, in this study, two moving magnets as shown in the equivalent model, Figure 2. The system of two identical center masses is modeled using two degrees of freedom (dofs) Duffing equations as shown in the system of equations below:

$\left\{\begin{array}{l}\ddot{a_{1}}+c_{t} \dot{a_{1}}+\omega_{0}^{2}\left[(1+2 \beta) a_{1}-\beta a_{2}\right]+f_{n l} a_{1}^{3}=(1+g) \ddot{Y} \\ \ddot{a_{2}}+c_{t} \dot{a_{2}}+\omega_{0}^{2}\left[(1+2 \beta) a_{2}-\beta a_{1}\right]+f_{n l} a_{2}^{3}=(1+g) \ddot{Y}\end{array}\right.$

$$
c_{t}=2 \xi \omega_{0} m, \omega_{0}^{2}=\frac{k_{m e c}^{l}}{m}, \beta=\frac{k_{m e c}^{l}}{k_{m g}}, f_{n l}=\frac{k_{m e c}^{n l}}{m}
$$

Where $a_{1}$ and $a_{2}$ are the generalized coordinates, $c_{t}$ is the total damping factor which is the sum of mechanical $c$ and $c_{e}$ electrical damping, $\omega_{0}$ is the eigenfrequency of each decoupled dof, $m$ is the equivalent mass, $\beta$ is the coupling coefficient, $k_{m e c}^{l}$ is the linear mechanical stiffness, $k_{m e c}^{n l}$ is the nonlinear mechanical stiffness, $f_{n l}$ is the nonlinear term which contains only the mechanical nonlinearity resulting from the large displacements of the beams, $g$ is the mass ratio and $\ddot{Y}$ is the acceleration of the basis excitation as shown in Figure 2.

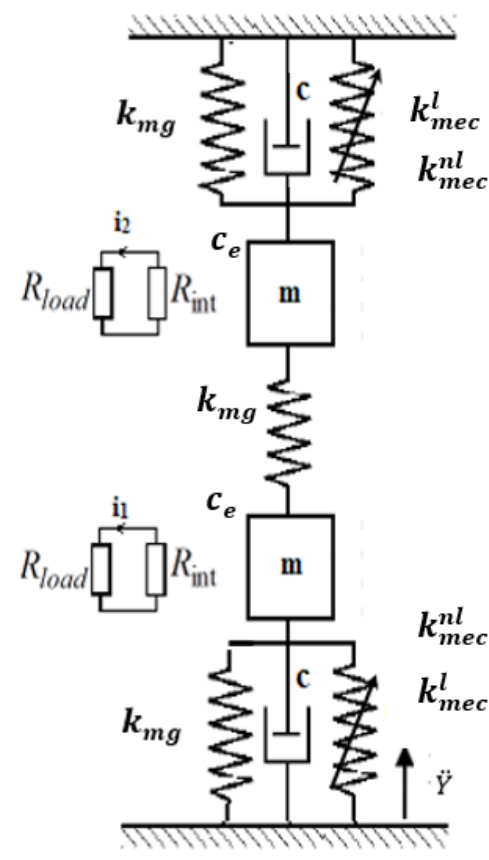

Figure 2. The equivalent two dof model of the VEH

The parameters shown in Figure 2 are $R_{\text {load }}$ and $R_{\text {int }}$ the load and internal resistances of the coil attached to the load circuit for energy harvesting.

It is assumed that the magnetic nonlinearity is neglected compared to the mechanical nonlinearity in the case of a weak coupling between the two beams.

\section{Results and discussion}

The frequency responses of the two dofs are obtained by solving the system of equations using the finite difference method. The continuation procedure was achieved by the arc-length method.

As shown in Figure 3 , the amplitude of the frequency response increases significantly in the nonlinear case compared to the linear one. In addition, we notice that the bandwidth (BW2) after introducing the nonlinearity is enlarged by $45 \%$ compared to the bandwidth (BW1) in the linear case.

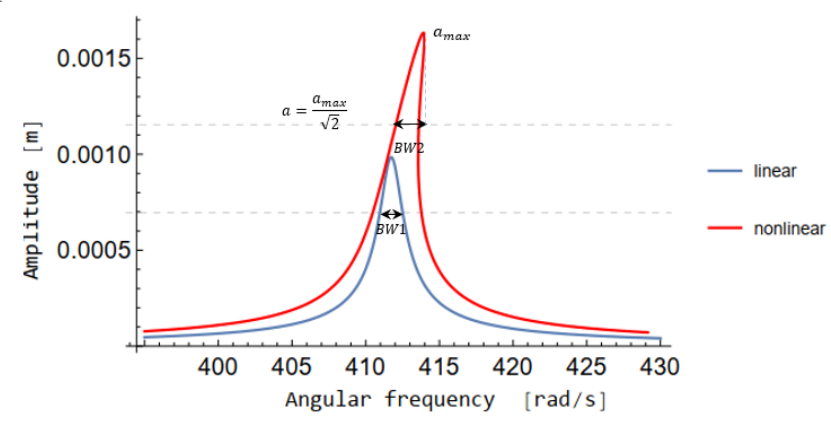

Figure 3. Linear and nonlinear frequency responses of the VEH

The performed simulations of these frequency responses for linear and nonlinear cases are evaluated with accelerations of a basis excitation respectively equal to $0.6 \mathrm{~g}$ and $1 g$.

The average harvested power is expressed as follows:

$$
P=R_{\text {load }}\left(\frac{\delta \omega_{0}}{R_{\text {int }}+R_{\text {load }}}\right)^{2}\left[a_{1 \max }^{2}+a_{2 \max }^{2}\right]
$$

Where $\delta$ is the electromagnetic coefficient defined as the variation of the magnetic field through the coil.

The numerical value of this coefficient is estimated by FEMM software and is equal to $1.04 \mathrm{Vs} \mathrm{m}^{-1}$.

With $R_{\text {load }}=12 \Omega$, the harvested power is equal to $24 m W$.

\subsection{Effects of mistuning}

In the case of mass mistuning, the system governing the proposed VEH can be expressed as follows: 
$\left\{\begin{array}{c}\ddot{a_{1}}+c_{t} \dot{a_{1}}+\omega_{0}^{2}\left[(1+2 \beta) a_{1}-\beta a_{2}\right]+f_{n l} a_{1}^{3}=(1+g) \ddot{Y}, \\ \alpha \ddot{a_{2}}+c_{t} \dot{a_{2}}+\omega_{0}^{2}\left[(1+2 \beta) a_{2}-\beta a_{1}\right]+f_{n l} a_{2}^{3}=(\alpha+g) \ddot{Y}\end{array}\right.$

where $\alpha$ is the mass mistuning coefficient. It represents the ratio between the masses of the unperturbed moving magnet to the unperturbed one.

Figure 4 illustrates the frequency response for nonlinear periodic and quasi-periodic structures with $\beta=0.008$, an acceleration equal to $1 \mathrm{~g}$ and a mistuning $\alpha=1.01$. As depicted in this figure, the introduction of mistuning in the studied system improves the bandwidth. We can notice that BW3 is higher than BW2 (Figures 3 and 4). The bandwidth is increased by $25 \%$ compared to the nonlinear case without mistuning and by $83 \%$ compared to the linear case.

On the other hand, the amplitude of the perturbed dof was increased compared to the amplitude of the unperturbed dof. This phenomenon, called energy localization, can be considered as a source of energy enhancement.

With $\alpha=1.01$ and $R_{\text {load }}=12 \Omega$, the harvested power, in this case, is equal to $30 \mathrm{~mW}$. The harvested power is enhanced by $25 \%$ compared to the results of periodic and quasi-periodic systems.

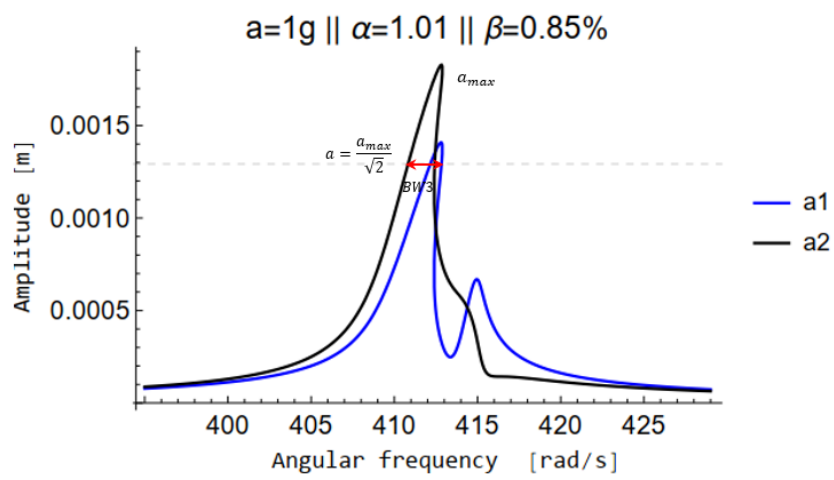

Figure 4. Effect of mistuning

\section{Conclusions}

In this paper, a multimodal vibration electromagnetic harvester has been studied. The mechanical nonlinearity introduced in the periodic system enhances the amplitude of vibration and enlarges the bandwidth as well. Mass mistuning technique was also considered. Its effect on frequency response as well on the harvested power was investigated. The obtained results show that the mass perturbation of one of the moving magnets increases more the amplitude and the bandwidth of the quasi-periodic system. Thus, theses aspects can be exploited in a way to improve the harvested power from a VEH.

\section{References}

[1] B. Yang, G. Lee, W. Xiang, J. Xie, J. Han He, R. K. Koltanka, S. P. Low and H. Feng, Electromagnetic energy harvesting from vibrations of multiple frequencies. Journal of Micromechanics and Microengineering, 19(3): 035001, 2009.

[2] I. Abed, N. Kacem, N. Bouhaddi, M.L. Bouazizi, Multi-modal vibration energy harvesting approach based on nonlinear oscillator arrays under magnetic levitation, Smart Materials and Structures, 2016.

[3] Y. Tadesse, S. Zhang and S. Priya, Multimodal energy harvesting system: Piezoelectric and electromagnetic,Journal of Intelligent Material Systems and Structures, 20(5):625-632, 2009.

[4] S. Mahmoudi, N. Kacem and N.Bouhaddi,Enhancement of the performance of a hybrid nonlinear vibration energy harvester based on piezoelectric and electromagnetic transductions, Smart Materials and Structures, 2014.

[5] Z. Zergoune, N. Kacem and N. Bouhaddi, Vibration energy localization from nonlinear quasi-periodic coupled magnets, Advences in Acoustics and Vibration II.ICAV 2018. Applied condition Monitoring vol 13. Springer, Cham.

[6] PV. Malaji and S.F. AliEnergy harvesting from near periodic structures, Vib. Eng. Technol. Mach., 2015.

[7] B.P. Mann, N.D. Sims Energy harvesting from the nonlinear oscillations of magnetic levitation, J. Sound Vib,319, 515530, 2009. 